\title{
O uso de jogos e desafios no ensino de lógica matemática
}

\author{
Ana Cecilia Togni* \\ Traducción: Óscar Torres Angarita
}

Fecha de elaboración: abril 28 de 2005

Fecha de aceptación: mayo 20 de 2005

Resumen. Este artículo es resultado de mi trabajo como profesora de la asignatura Lógica Matemática en la carrera de formación de maestros y de mi inquietud sobre cómo se ha impartido hasta ahora con estudiantes que ya son maestros, debido al modo muy formal de manejarla que deja huecos en la construcción de su pensamiento lógico inductivo y deductivo. Se cree que la metodología trabajada ayuda a superar esta omisión.

Palabras clave: lógica matemática, juegos, desafíos, inducción, deducción.

Resumo. Este artigo, resulta do meu trabalho como professora da disciplina de Lógica Matemática em cursos de formação de professores em nível de graduação e é também resultado da minha preocupação com a forma como essa disciplina vem sendo ministrada aos alunos-professores, ao longo do tempo, ou seja de uma maneira

Introdução. Atualmente, vive-se uma época, onde a tecnologia e a eletrônica avançam rapidamente, ou seja em pouco tempo os instrumentos e aparelhos que hoje se considera de última geração estarão ultrapassados, então a pergunta que se pode fazer é a seguinte:

- O que é preciso para que se possa enfrentar estas constantes e rápidas mudanças?

A resposta para esta questão é que as pessoas tenham pensamento lógico.

* Licenciada en Matemáticas por la Universidad Federal de Rio Grande do Sul; posgrado en Matemáticas por la Universidad de Santa Cruz do Sul; maestría en Educación por la Universidad de Vale do Rio dos Sinos-São Leopoldo-Rs; muito formal, deixando espaços na construção de um pensamento lógico, seja ele indutivo ou dedutivo. Creio que a forma que encontrei para trabalhar com a referida disciplina, procura suprir esta lacuna.

Palaviras chave: lógica matemática, jogos, desafios, indução, dedução.

Summary. This paper is a result of my work as a professor of Logical Mathematics in teaching of undergraduate students. It is a direct result of my concern of how this discipline develops in classes. Due to its very formal approach there have been gaps in the way the logical and deductive way of thinking are being developed among students. It is believed that an elaborated methodology helps overcome this discrepancy.

Key words: logical mathematics, games, challenge, induction, deduction.

Introducción. En la época actual la tecnología y la electrónica avanzan con rapidez y en corto tiempo los instrumentos y los equipos que hoy se consideran de última generación serán obsoletos. Por tanto, surge la pregunta:

- ¿Qué se necesita para enfrentar estos cambios constantes y rápidos?

La respuesta es que las personas requieren tener un pensamiento lógico.

doctoranda en Informática en la Educación por la Universidad Federal de Rio Grande do Sul; profesora de Matemáticas en el Centro Universitario Univates en Lajeado-Rio Grande do Sul. Red Rie-Brasil. chica@certelnet.com.br 
Volta-se a perguntar:

- De que forma se pode construir este pensamento?

A resposta desta questão deve ser: estudando Lógica.

Então, pergunta-se: O que é Lógica?

Se consultados livros sobre Lógica ou dicionários se encontram diversas definições tais como:

- "Lógica é a ciência que tem por objeto o estudo das leis do raciocínio; coerência; raciocínio encadeado; ligação de idéias". (Fernandes, Luft, Guimarães)

- "A Lógica formal é uma ciência que determina as formas corretas (ou válidas) de raciocínio". (Dopp)

"Lógica é a ciência das formas de pensamento". (Liard)

"Lógica é a linguagem que estrutura as linguagens descritivas". (Hegenberg)

- "Lógica é a ciência da argumentação, enquanto esta é diretiva da operação de raciocinar". (Telles júnior)

" "Lógica é a arte que dirige o próprio ato da razão, isto é que nos permite chegar com ordem facilmente e sem erro, ao próprio ato da razão". (Maritain)

- "O estudo da Lógica é o estudo dos métodos e princípios usados para distinguir o raciocínio correto do incorreto". (Coppi)

Como se pode perceber existe uma diversidade de concepções, porém resumidamente todas se referem a que a Lógica tem como objeto de estudo as formas de pensamento, de suas leis e de seus princípios.

De outra parte, como é uma ciência que opera com "formas despidas de conteúdo" (Bastos, Keller 1998) muitas vezes sente-se uma sensação de não utilização por isso seu estudo torna-se tedioso, cansativo e com muita dificuldade.

Este é um dos motivos pelos quais já de há muito tempo, tenho me preocupado em como trabalhar com a disciplina de Lógica Matemática nos cursos de formação de professores, em nível de $3^{\circ}$ grau, uma vez que ela em geral é ministrada de uma forma muito formal e não propicia aos alunos a inter-relação necessária com outras disciplinas do curso. A partir desta preocupação e não deixando de lado a ementa e o programa estabelecido para este componente curricular, procurei há alguns semestres atrás estabele-
Entonces surge otra pregunta:

-¿Cómo puede construirse esta clase de pensamiento? La respuesta debe ser: estudiando lógica. Viene luego una tercera pregunta: ¿qué es la lógica?

En los libros y en los diccionarios se encuentran diferentes definiciones de lógica; por ejemplo:

- "Lógica es la ciencia que tiene por objeto el estudio de las leyes del raciocinio; coherencia; raciocinio articulado; ligazón de ideas". (Fernandes, Luft, Guimarães)

- "La lógica formal es una ciencia que determina las formas correctas (o válidas) de raciocinio". (Dopp)

- "Lógica es la ciencia de las formas del pensamiento". (Liard)

- "Lógica es un lenguaje que estructura los lenguajes descriptivos". (Hegenberg)

"Lógica es la ciencia de la argumentación en cuanto ésta dirige la operación de razonar". (Teller jr.)

- "Lógica es el arte que dirige el propio acto de la razón, es decir, que nos permite llegar fácilmente, con orden y sin error, al propio acto de la razón". (Maritain)

- "El estudio de la lógica es el estudio de los métodos y principios utilizados para distinguir un raciocinio correcto de uno incorrecto". (Coppi)

Como puede percibirse, existen diversos conceptos de lógica, pero en resumen todos se refieren a que la lógica tiene como objeto de estudio las formas de pensamiento, sus leyes y sus principios.

Por otra parte, como es una ciencia que opera con "formas desprovistas de contenido" (Bastos, Keller, 1998), con frecuencia se tiene la sensación de que se trata de algo inútil y por eso estudiarla se vuelve tedioso, agotador y muy difícil.

Este es uno de los motivos por los cuales desde hace mucho tiempo me preocupa cómo trabajar con la Lógica Matemática en los cursos de formación de profesores, en grado tercero, después de que en general la lógica ya ha sido vista de una manera muy formal e inapropiada para que los alumnos la interrelacionen con otras materias del curso. A partir de esta preocupación, y sin dejar de lado el plan y el programa establecidos para este componente curricular, hace unos 
cer uma metodologia de ensino que pudesse atender simultaneamente a estes itens, bem como tornasse as aulas mais interessantes e participativas.

\section{Reflexões iniciais}

Com a finalidade de realizar este intento, uma vez que meu objetivo primordial é nesta disciplina possibilitar que os alunos aprendam a realizar demonstrações e deduzir fórmulas foi realizar a mim mesma alguns questionamentos tais como:

a) Que conhecimentos tem meus alunos?

b) Sendo grande parte deles professores em escolas de ensino fundamental e médio, como trabalham eles com seus alunos?

c) Que técnicas pedagógicas utilizar?

d) Como utilizar essas técnicas com referencia aos conteúdos programados para a disciplina?

e) Como realizar a avaliação desses alunos, uma vez que no nosso sistema de ensino notas são necessárias?

Refletindo sobre estes questionamentos iniciei a preparação das atividades do semestre, levando em conta que a cada turma tem sua bagagem de conhecimentos e que ao se empregar alguma técnica pedagógica, os envolvidos nesta aplicação estarão normalmente trabalhando em grupos, cujo número de componentes diferirá em função do número de alunos que cursam a disciplina. È necessário ainda levar em conta que entre outras coisas segundo Antunes (1990) que:

a) o conhecimento é obtido através de fatos e experiências;

b) o conhecimento não deve contradizer experiências e fatos comprovados;

c) um conhecimento se justifica quando parte de uma experiência é evidenciada por outro conhecimento.

Após estas reflexões optei por realizar atividades que envolvessem aulas expositivo-dialogadas entremeadas por diversas técnicas que possibilitassem o alcance do objetivo primordial proposto. Entre estas utilizaram -se: jogos, desafios lógicos, quebra-cabeças, fluxogra- semestres traté de establecer una metodología de enseñanza que pueda atender simultáneamente estos aspectos y que también haga que el trabajo en las aulas se vuelva más interesante y participativo.

\section{Reflexiones iniciales}

Con el fin de llevar a la práctica mi propuesta, y teniendo en cuenta que mi objetivo primordial es que los estudiantes aprendan a hacer demostraciones y a deducir fórmulas, me hice preguntas como las siguientes:

a) ¿Cuáles son los conocimientos de mis alumnos?

b) Como gran parte de ellos son profesores en escuelas de enseñanza básica y media, ¿de qué manera trabajan con sus estudiantes?

c) ¿Qué técnicas pedagógicas usar?

d) ¿Cómo utilizar esas técnicas en relación con los contenidos programados para la disciplina?

e) ¿Cómo evaluar a los alumnos si se tiene en cuenta que en nuestro sistema educativo se evalúa con notas?

En medio de la reflexión sobre estas preguntas, inicié la preparación de las actividades del semestre, teniendo en cuenta que cada grupo de estudiantes tiene su bagaje de conocimientos y que al emplear alguna técnica pedagógica los implicados trabajarían normalmente en grupos cuya cantidad de integrantes dependerá del número de alumnos que cursan la materia. También es necesario tener en cuenta, entre otras cosas, que según Antunes (1990):

a) El conocimiento se obtiene a través de los hechos y las experiencias.

b) El conocimiento no debe contradecir las experiencias ni los hechos comprobados.

c) Un conocimiento se justifica cuando parte de una experiencia es evidenciada por otro conocimiento.

Después de estas reflexiones, opté por realizar actividades que involucraran clases expositivo-dialogadas, entremezcladas con diversas técnicas que posibilitasen el alcance del objetivo primordial propuesto. Algunas de las técnicas que se utilizaron fueron: juegos, desafíos lógicos, rompecabezas, diagramas de flujo, 
mas, noticias de jornal, confecção de pequenas resenhas etc, uma vez que segundo (Fischer: s.d.):

O objetivo principal de um programa de Matemática é ensinar os alunos a pensar. Nosso ensino deveria englobar aspectos principais do pensamento matemático, na medida que isto é possível. As atividades mais marcantes da do matemático são: a descoberta de demonstrações rigorosas e a construção de sistemas axiomáticos. Existem no entanto, outras atividades que, por deixarem menos sinais na obra acabada do matemático são, por isso, menos aparentes mas não menos importantes, tais como: reconhecer e extrair um conceito matemático de uma situação concreta; em seguida fazer várias formas de adivinhações, ou seja prever o resultado, prever as grandes linhas de uma demonstração antes de realiza-la, em detalhe. 'Adivinhar', assim compreendido, pode englobar generalizações a partir de casos observados, um raciocínio indutivo, uma argumentação por analogia, etc.

Dessa forma penso fica mais fácil para o aluno num primeiro momento "adivinhar" do que demonstrar com rigor, resolver problemas que envolvam situações concretas é mais agradável e menos desgastante do que resolver problemas utilizando estruturas que envolvam conceitos abstratos, pois normalmente o desencadeamento das idéias parte do concreto para o abstrato, da percepção para a conceituação e da conceituação para a simbologia.

\section{Como foram aplicadas as tècnicas?}

No primeiro encontro e após a apresentação, realizouse a explanação de como se conduziria a disciplina e a seguir para que se pudesse dar início as atividades, realizou-se uma discussão envolvendo Resolução de Problemas.

A primeira questão lançada foi:

-O que é para você um problema?

Após reflexões individuais e entre colegas anotou-se no quadro as respostas.

A pergunta seguinte foi: noticias periodísticas, elaboración de pequeñas reseñas, etc., pues según Fischer (s.d.):

El objetivo principal de un programa de matemáticas es enseñar a los alumnos a pensar. Nuestra enseñanza debería incluir los aspectos principales del pensamiento matemático, en la medida en que esto sea posible. Las actividades más importantes de un matemático o matemática son: un descubrimiento de demostraciones rigurosas y una construcción de sistemas axiomáticos. Sin embargo, existen otras actividades que, por dejar pocas huellas en el trabajo final de un matemático, son menos evidentes pero no menos importantes, tales como reconocer y extraer un concepto matemático de una situación concreta; después, poner en práctica varias formas de predicción, o sea prever un resultado, prever en detalle las grandes líneas de una demostración antes de realizarla. Así entendido, "adivinar" puede involucrar generalizaciones a partir de casos observados, un raciocinio inductivo, una argumentación por analogía, etc.

Pienso que de esa manera en un primer momento es más fácil para un alumno "adivinar" algo que demostrarlo con rigor, resolver problemas que impliquen situaciones concretas y más agradables y menos estresantes que resolver problemas utilizando estructuras que involucren conceptos abstractos, pues normalmente el desarrollo de las ideas va de lo concreto a lo abstracto, de la percepción a la conceptualización y de la conceptualización a la simbología.

\section{¿Cómo se aplicaron las técnicas?}

En el primer encuentro, después de la presentación se realizó una explicación de cómo se llevaría a cabo la enseñanza y luego para dar inicio a las actividades se planteó una discusión respecto a la resolución de problemas.

La primera pregunta fue:

-¿Qué considera usted un problema?

Después de las reflexiones individuales y entre los compañeros, se hizo un cuadro de respuestas.

La pregunta siguiente fue: 


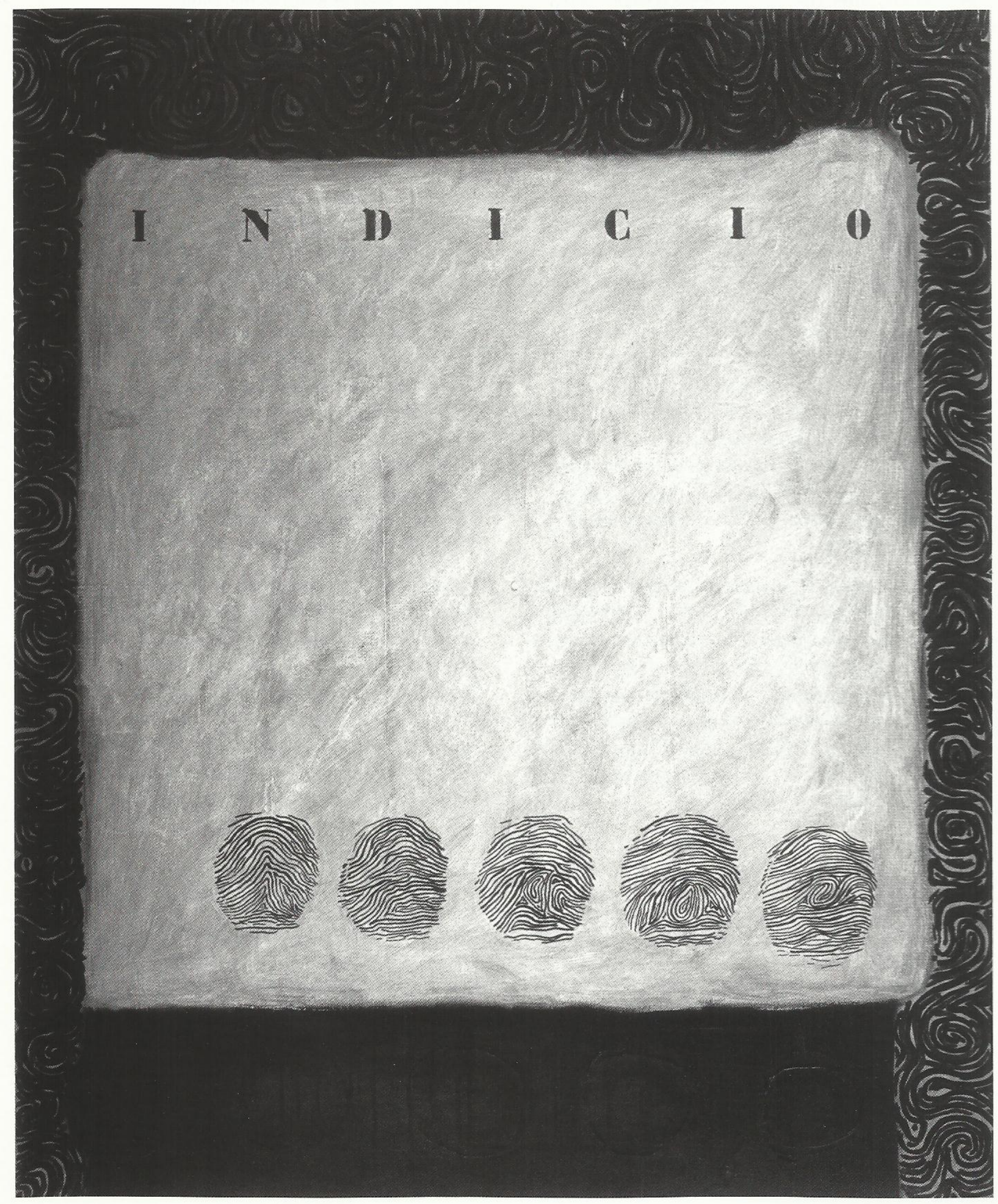


- O que é para você resolver um problema?

Da mesma forma, após reflexões individuais e entre os colegas anotou-se no quadro as respostas.

A partir dessas respostas, citou-se Polya (1945) que estabeleceu alguns passos para resolução de um problema. Para ele era necessário:

a) Compreender o problema.

b) Conceber um plano para resolução.

c) Executar este plano.

d) Realizar uma retrospectiva.

Quanto a como seguir estes passos citou-se Pozo e Echeverría (1998) que sugerem as seguintes perguntas para melhor compreender um problema:

- Qual a dificuldade do problema?

- Qual é a meta?

- Quais são os dados que estou usando como ponto de partida?

- Conheço algum problema similar?

Sugerem ainda:

- Reescrever o problema usando seus próprios termos.

- Explicar aos colegas em que consiste o problema.

- Modificar o formato da proposição do problema (usar gráficos, desenhos, etc.)

- Quando muito geral, concretizar o problema usando exemplos.

-Quando muito específico, tentar generalizar o problema.

De outra forma falou-se também em procedimentos heurísticos que podem ser utilizados para resolver problemas:

- Realizar tentativas por meio de ensaio e erro.

- Aplicar a análise, meios- fins.

- Dividir o problema em subproblemas.

- Estabelecer sub - metas.

- Decompor o problema.

- Procurar problemas análogos.

- Ir do conhecido ao desconhecido.

Trabalhe em sentido contrário da frente para trás.
- Para usted, ¿qué es resolver un problema?

De la misma forma, después de las reflexiones individuales y entre compañeros, se hizo otro cuadro de respuestas. A partir de éstas, se citó a Polya (1945), quien estableció algunos pasos para la resolución de un problema. Para él era necesario:

a) Comprender el problema.

b) Concebir un plan para la solución.

C) Poner en práctica este plan.

d) Realizar una retrospectiva.

Respecto a la manera de seguir estos pasos, se citó a Pozo y Echeverría (1998) que sugieren las preguntas siguientes para comprender mejor un problema:

- ¿Cuál es la dificultad del problema?

- ¿Cuál es la meta?

- ¿Cuáles son los datos que uso como punto de partida?

- ¿Conozco algún problema similar?

Esos autores también sugieren:

- Reescribir el problema usando términos propios.

- Explicar a los compañeros en qué consiste el problema.

- Modificar el formato de proposición del problema (usar gráficos, diseños, etc.)

- Cuando el problema sea muy general, concretarlo por medio de ejemplos.

- Cuando el problema sea muy específico, tratar de generalizarlo.

Asimismo, se habló de procedimientos heurísticos que pueden utilizarse para resolver problemas:

- Hacer intentos mediante el ensayo y el error.

- Aplicar el análisis medios-fines.

- Dividir el problema en subproblemas.

- Establecer metas intermedias.

- Descomponer el problema.

- Pensar en problemas similares.

- Ir de lo conocido a lo desconocido.

- Trabajar en sentido contrario: de adelante hacia atrás. 
A seguir falou-se em tipos de problemas e apresentaram-se problemas determinação e problemas de demonstração.

Nos problemas de determinação o objetivo primeiro é encontrar a incógnita do problema, eles podem ser teóricos ou práticos, abstratos ou concretos, podem também ser problemas sérios ou simplesmente enigmas e charadas. As partes principais deste tipo de problemas são: a incógnita, os dados e o condicionante.

Já nos casos dos problemas de demonstração, o objetivo é mostrar conclusivamente que certa afirmativa, claramente enunciada, é verdadeira ou é falsa. Para isso deve-se provar uma ou outra destas condições. Problemas de demonstração em geral são teoremas e suas partes principais são a hipótese e a conclusão. Deve-se observar porém que nem todos os teoremas podem ser naturalmente divididos dessa forma.

A partir dessas observações iniciou-se a aplicação de cada uma das técnicas já citadas, sendo uma por aula, pois o semestre conta com 16 (dezesseis) encontros em sala de aula.

a) A primeira delas foi à divisão da turma em grupos e a distribuição pelo professor de uma atividade denominada: Jogos Boole-Histórias Lógicas, formada por pequenas histórias narradas em forma de poema, acompanhadas por cartas ilustrativas, que deveriam ser colocadas na ordem da história. Depois da realização da atividade pelo grupo, as histórias eram trocadas entre os mesmos. A seguir foi solicitado pelo professor que cada grupo construísse uma história daquele tipo.

b) A segunda atividade foi à realização de desafios lógicos, desafios esses nos quais são dadas pistas diversas para que se encontrem as respostas. Também, cada grupo, após resolver alguns desses deveria criar pelo menos um. Alguns grupos criaram mais de um.

c) O uso de Fluxogramas (Diagramas de Fluxo) também tornou - se uma atividade interessante quando se trabalhou com álgebra. Aqui resolveram -se equações, inequações, verificaram-se, identidades entre outras atividades
Después se habló de tipos de problemas y se presentaron problemas de determinación y problemas de demostración.

En los problemas de determinación el primer objetivo es encontrar la incógnita del problema; estos problemas pueden ser teóricos o prácticos, abstractos o concretos y también pueden ser problemas serios o simplemente enigmas y acertijos. Las partes principales de este tipo de problemas son: la incógnita, los datos y la condición.

En los casos de los problemas de demostración, el objetivo es mostrar de forma conclusiva que una afirmación, enunciada con claridad, es verdadera o falsa, para lo cual se debe probar una u otra de estas condiciones. En general, los problemas de demostración son teoremas, cuyas partes principales son la hipótesis y la conclusión. Debe observarse, sin embargo, que no todos los teoremas pueden dividirse de esa manera.

A partir de esas observaciones se inició la aplicación de cada una de las técnicas citadas, una por clase, pues el semestre consta de 16 clases.

a) La primera técnica consistió en dividir la clase en grupos; el profesor distribuyó una actividad denominada Juegos Boleanos-Historias lógicas, formada por pequeñas narraciones en forma de poema, acompañadas por ilustraciones que deberían ser colocadas en el orden de la historia. Después de que los grupos realiza la actividad, se intercambiaban las historias. Enseguida el profesor solicitaba que cada grupo elaborara una historia del mismo tipo.

b) La segunda actividad fue la realización de desafíos lógicos, en los cuales, a partir de unas pistas, había que encontrar las respuestas. Del mismo modo, después de que cada grupo resolviera algunos de esos problemas, debía crear por lo menos uno similar. Algunos grupos crearon más de uno.

c) El uso de diagramas de flujo también se volvió una actividad interesante cuando se trabajó con álgebra. Entre otras actividades, se resolvieron ecuaciones, desigualdades, que luego se verificaron, e identidades. 
d) Observação de jogos de mesa, tais como: dama, trilha (moinho), jogos de baralho, xadrez, bingo, etc., e jogos infantis, os quais podem ser jogados na rua como por exemplo A amarelinha. Deveriam observar suas regras e como se joga, e, por este meio verificar o que de matemática existe neles e a partir destas conclusões partiu-se para a formalização demonstrando as fórmulas em cada caso, quando necessário. (Alguns dos assuntos que apareceram: Progressões Aritméticas e Geométricas, Análise Combinatória, números antecessores e sucessivos, pares e ímpares, etc.).

e) Análise de embalagens de produtos comercializados em lojas e supermercados, onde aparecem erros lógico-matemáticos nas recomendações do fabricante. (Recomendações sobre acondicionamento, relativas à temperatura, ou aos ingredientes que compõe o referido produto).

f) Leitura de notícias de jornal e delas extrair dados para execução de problemas, antes porém comentar a mesma na sala de aula.

g) Escolher um assunto de matemática ministrado em classes do ensino fundamental e médio e desenvolve-lo num pequeno texto de no máximo 25 linhas, que depois foi analisado por um colega da sala de aula. (Estas duas últimas atividades visam desenvolver a expressão oral e escrita, pois, jovens professores apresentam muitas vezes dificuldade neste sentido).

h) Demonstrações de teoremas, apresentados algumas vezes em forma de atividades concretas como por exemplo o Teorema de Pick; ou dadas outras atividades de demonstração através de "brincadeiras geométricas" por exemplo para que usem o método da Indução e da Dedução.

i) Resolução de jogos eletrônicos como por exemplo "Torre de Hanói" no computador, e a descoberta da lei que coordena a troca dos discos de uma haste para outra, o que também motiva o uso do método indutivo ou dedutivo conforme o caso.

Deve-se salientar que embora realizando todas estas atividades práticas, não se descuidou da parte formal do conteúdo a ser desenvolvido, uma vez que elas, realizadas individualmente ou em grupo direcionavam para a formalização. d) Observación de juegos de mesa, tales como damas, molino, juego de baraja, ajedrez, bingo, etc., y juegos infantiles que pueden practicarse en la calle, por ejemplo "A amarelinha". Debían tenerse en cuenta sus reglas y el modo de jugarlos, y de esta forma verificar qué aspectos de las matemáticas existen en ellos; estas conclusiones fueron la base para la formalización mediante la demostración de las fórmulas en cada caso cuando era necesario. (Los siguientes son algunos de los temas que surgieron: progresiones aritméticas y geométricas, análisis combinatorio, números antecesores y sucesores, pares e impares, etc.).

e) Análisis de empaques de productos comercializados en tiendas y supermercados, en los cuales aparecen errores lógico-matemáticos en las recomendaciones del fabricante (recomendaciones en cuanto a condiciones de conservación, temperatura, o relacionadas con los ingredientes de los productos).

f) Lectura de noticias periodísticas que, después de comentarlas en clase, se utilizaban para extraer datos y crear problemas.

g) Escoger un tema de matemáticas visto en las clases de enseñanza básica y media y desarrollarlo en un texto de máximo 25 líneas, que después era analizado por un compañero de la clase. (Las dos últimas actividades se dirigían a desarrollar la expresión oral y escrita, debido a que a menudo los profesores jóvenes tienen dificultades en esta área).

h) Demostraciones de teoremas que se presentaban algunas veces en forma de actividades concretas, como el teorema de Pick; o se daban otras actividades de demostración, por ejemplo a través de "diversiones geométricas" para que usaran el método de inducción y deducción.

i) Resolución de juegos electrónicos en computador, por ejemplo "Torre de Hanoi", y el descubrimiento de la ley que coordina el paso de platillos entre un palo y otro, lo que también estimula el uso del método inductivo o deductivo, según el caso.

Debe resaltarse que, aun cuando se realizaron todas estas actividades prácticas, no se descuidó la parte formal del contenido que se iba a desarrollar, pues las actividades individuales o grupales se dirigían a la formalización. 


\section{Conclusões}

Pelo que tenho tido oportunidade de colher junto aos meus alunos nos últimos semestres, acredito que estou alcançando o objetivo a que me propus ou seja trabalhar com Lógica Matemática de forma a possibilitar sua utilização em outras disciplinas do curso de Licenciatura, sem torná-la uma disciplina de difícil entendimento.

\section{Referências}

COPPI, Irving M. 1978. Introdução à lógica. $2^{a}$ ed. São Paulo: Mestre Jou.

D'AMORE, Bruno. 2001. Problemas. Pedagogia y psicologia de la matemática em la actividade de resolucion de problemas. Madrid: Editorial Síntesis.

DOPP, Joseph. 1970. Noções de lógica formal. São Paulo: Herder.

FERNANDES, Francisco, LUFT, Pedro y GUIMARÃES, F..M. 1992. Dicionário brasileiro Globo. 24aㅗ ed. São Paulo: Globo.

FISCHER, Maria C. Uma reflexão sobre o ensino da matemática. A lógica e as demonstrações matemáticas. (Multicopiado)

\section{Diálogo del conocimiento}

El artículo muestra cómo aumenta el interés en cambiar la metodología tradicional de enseñanza de la lógica matemáti$\mathrm{ca}$, que normalmente se imparte en las aulas universitarias, máxime si se está encaminado a la formación de maestros. Es importante notar en el texto que la reflexión sobre la corresponsabilidad del docente en la construcción de un pensamiento lógico inductivo y deductivo en los estudiantes, genera propuestas como la de la profesora Togni, y que se pueden hacer grandes aportes a subsanar en parte estas falencias, por lo menos en lo que corresponde al docente.

Cada una de las técnicas aporta de manera ágil la forma de llevar al estudiante de lo concreto a lo abstracto, y en esta variedad se nota la recursividad de los docentes para mostrar que es posible desarrollar las matemáticas en actividades diferentes a la formalidad propia de un aula de clase. Esto hace ver la posibilidad de generar aprendizaje de una manera más significativa para el estudiante y de agilizar la enseñanza de la lógica de manera práctica y recursiva, mediante el diseño de estrategias que involucren al estudiante para que su aprendizaje sea más participativo.

Sin embargo, la información puede enriquecerse si: el marco teórico se explicita mejor con la propuesta; se presentan los

\section{Conclusiones}

Por lo que he tenido oportunidad de comprobar con mis alumnos de los últimos semestres, creo que estoy alcanzando el objetivo que me propuse, esto es, trabajar con la lógica matemática de manera que se posibilite su utilización en otras materias de la licenciatura, sin volverla una disciplina difícil de entender.

HEGENBERG, Leônidas. 1966. Lógica simbólica. São Paulo: Herder. LIARD, L. Lógica. 1965. 6ª ed. São Paulo: Cia. Editora Nacional.

MARITAIN, Jacques. 1980. Elementos de filosofia II: a ordem dos conceitos, lógica menor. Rio de Janeiro: Agir.

POLYA, George. 1995. A arte de resolver problemas. $2^{\text {a }}$ reimp. Rio de Janeiro: Interciência.

POZO, Juan I. (comp.). 1998. A solução de problemas. Aprender a resolver, resolver para aprender. Porto Alegre: Artes Médicas Sul Ltda.

resultados de algunas soluciones a las preguntas iniciales que se plantearon, y de ser posible se contrastan con el marco teórico o con la misma propuesta; se dan a conocer las tablas que se mencionan sobre los resultados del grupo acerca de la resolución de problemas; se comenta la forma de aplicación de las técnicas; se presentan y analizan los resultados de las nueve técnicas desarrolladas en clase, y se amplían las conclusiones con los ítems anteriores. Además, sería un gran aporte que se ubique a los lectores sobre el entorno del aula de clase, como número de estudiantes, tiempo de cada sesión, dedicación a cada actividad, frecuencia de aplicación de la estrategia, entre otros. Para los docentes que comparten estas inquietudes sería conveniente reconocer el camino ya recorrido y poder implementar o ajustar una o todas las técnicas, no necesariamente para estudiantes de licenciatura, sino para todos los estudiantes, con el objetivo de contribuir a la construcción de un pensamiento formal.

Desde luego, existe la opción de la comunicación directa con la profesora Togni a fin de establecer un diálogo sobre estos y otros temas de su propuesta de trabajo.

Nina Stella Clavijo Vargas 